\title{
Oligonucleotide-mediated genetic transformation of Borrelia burgdorferi
}

\author{
D. Scott Samuels ${ }^{1,2}$ and Claude F. Garon ${ }^{2}$ \\ Author for correspondence: Scott Samuels. Tel: +1 4062436145 . Fax: + 14062434184. \\ e-mail: samuels@selway.umt.edu
}

1 Division of Biological Sciences, University of Montana, Missoula, Montana 59812, USA

2 Bacterial Pathogenesis Section, Rocky Mountain Laboratories Microscopy Branch, National Institute of Allergy and Infectious Diseases, Hamilton, Montana 59840, USA

\begin{abstract}
We have used short oligonucleotides to genetically transform the Lyme disease spirochaete Borrelia burgdorferi. The oligonucleotides are derived from the sequence of an Arg-133 to lle mutant gyrB (chromosomal) gene that confers resistance to the antibiotic coumermycin $A_{1}$. Oligonucleotides were about 10000 -fold less efficient at transformation, on a molar basis, than longer PCR-generated substrates. All of the transformants tested contained the predicted site-directed silent mutation in their gyrB genes. Antisense oligonucleotides were more efficient at transformation than either sense or double-stranded oligonucleotides. This is the first demonstration of oligonucleotides used to introduce site-directed mutations directly into the genome of a bacterium.
\end{abstract}

Keywords: Lyme disease, Borrelia burgdorferi, oligonucleotide, genetic transformation, electroporation, gyrB

\section{INTRODUCTION}

Borrelia burgdorferi, a causative agent of Lyme disease (Baranton \& Old, 1995; Barbour \& Fish, 1993; Steere, 1994), is a spirochaete with a genome that consists of both linear and circular plasmids as well as a linear chromosome (Hinnebusch \& Tilly, 1993; Saint Girons et al., 1994). B. burgdorferi is highly susceptible to the coumarin antibiotic coumermycin $A_{1}$ (Samuels \& Garon, 1993), which targets the B subunit of DNA gyrase (Maxwell, 1993). We have isolated and characterized spontaneous mutants of $B$. burgdorferi that are resistant to coumermycin $A_{1}$ (Samuels et al., 1994b). Currently, these are the only available mutants of $B$. burgdorferi resistant to an antimicrobial agent. We previously reported on two classes of coumermycin $A_{1}$ resistant mutants that had single point mutations in their gyrB genes that converted Arg-133 to either Gly or Ile (Samuels et al., 1994b). These mutant genes have been used as selectable markers for the first demonstration of genetic transfer in any species of Borrelia (Samuels et al., 1994a). Successful transformation was confirmed using PCR to create site-directed mutant gyr $B$ genes and introducing them into wild-type cells by electroporation. Recently, we have inserted a coumermycin $\mathrm{A}_{1}$-resistant gyrB gene cassette into the $26 \mathrm{~kb}$ circular plasmid of B. burgdorferi (Rosa et al., 1996).
Electroporation, the application of a high voltage electrical pulse to disrupt cell membranes, has been used to genetically transform a variety of micro-organisms (Nickoloff, 1995; Shigekawa \& Dower, 1988; Trevors et al., 1992). We have previously electroporated PCRgenerated 840 bp DNA substrates into $B$. burgdorferi and selected for the homologous recombination of these DNA molecules into the chromosome (Samuels et al., 1994a). The yeast Saccharomyces cerevisiae can be transformed with short oligonucleotides (Moerschell et al., 1988). We now demonstrate that single-stranded oligonucleotides of 27 bases can be used to introduce site-directed mutations conferring coumarin resistance into the gyrB gene of $B$. burgdorferi.

\section{METHODS}

DNA. Oligonucleotides 385F/398T399T (5' GTTTATGTTAATATTGATGGAAAAATT $3^{\prime}, M_{\mathrm{r}}$ 8834) and 411R/ 398A399A (5' AATTTTTCCATCAATATTAACATAAAC $\left.3^{\prime}, M_{r} 8652\right)$ were used as transformation substrates. These oligonucleotides are inverse complements of each other and were used previously to create site-directed mutations (Samuels et al., 1994a). They encode amino acids 129-137 of the $B$. burgdorferi strain B31 gyrB gene product and include two point mutations that change the Arg-133 AGA codon to an ATT Ile codon. The double-stranded oligonucleotide transformation substrate was generated by boiling $2.5 \mathrm{mg}$ $\mathrm{ml}^{-1}\left(\sim 0.3 \mu \mathrm{mol} \mathrm{ml} l^{-1}\right)$ each of $385 \mathrm{~F} / 398 \mathrm{~T} 399 \mathrm{~T}$ and $411 \mathrm{R}$ / 398A $399 \mathrm{~A}$ together for $1 \mathrm{~min}$ and cooling slowly to allow for annealing $\left(100^{\circ} \mathrm{C}-65^{\circ} \mathrm{C}\right.$ in $\sim 10 \mathrm{~min}$ and $65^{\circ} \mathrm{C}-25^{\circ} \mathrm{C}$ in $\sim 60 \mathrm{~min})$. 
Electroporation. Wild-type B. burgdorferi cells were made competent for electrotransformation as described previously (Samuels, 1995; Samuels et al., 1994a). Briefly, cells were grown to $\sim 5 \times 10^{7}$ cells $\mathrm{ml}^{-1}$, washed twice in Dulbecco's phosphate-buffered saline and three times in $272 \mathrm{mM}$ sucrose and $15 \%(\mathrm{v} / \mathrm{v})$ glycerol (EPS), and resuspended in EPS at $\sim 10^{10}$ cells $\mathrm{ml}^{-1}$. Cells were mixed with $5-10 \mu \mathrm{g}$ $(570-1140 \mathrm{pmol})$ oligonucleotide or $0.5-1.5 \mu \mathrm{g}(1-3 \mathrm{pmol})$ of an $840 \mathrm{bp}$ PCR product, transferred to a $0.2 \mathrm{~cm}$ electroporation cuvette (BTX), and subjected to a single pulse of $2.5 \mathrm{kV}, 25 \mu \mathrm{F}$ and $200 \Omega$ from a Gene Pulser with Pulse Controller (Bio-Rad).

Electron microscopy. Fractions of competent cells were removed from the cuvette both before and immediately after electroporation and applied to the surface of a parlodioncoated grid, negatively stained for $30 \mathrm{~s}$ with a $2 \%(w / v)$ ammonium molybdate solution, air-dried and examined in a Philips CM10 transmission electron microscope at $80 \mathrm{kV}$ accelerating voltage.

Selection and analysis of transformants. Cells were recovered in $10 \mathrm{ml}$ of Barbour-Stoenner-Kelly II (BSK II) medium without antibiotics for $19-25 \mathrm{~h}$ at $32^{\circ} \mathrm{C}$ and plated in solid medium containing $0.2 \mu \mathrm{g}$ coumermycin $\mathrm{A}_{1} \mathrm{ml}^{-1}$ as described previously (Rosa \& Hogan, 1992; Samuels, 1995; Samuels et al., 1994b). Individual clones were expanded and DNA was isolated from each clone. A portion of the gyrB gene was then amplified from each clone by PCR and sequenced using a dsDNA Cycle Sequencing System (Life Technologies) as described previously (Samuels et al., 1994b).

\section{RESULTS}

The structural effects of a single electric field pulse on $B$. burgdorferi are shown in Fig. 1(b). The darkly stained regions, suggesting marked cell surface disruptions, are seen along the entire length of the spirochaete. Although the number of these darkly stained regions per cell appears to vary broadly, most of the spirochaetes in a field appear to have them. These surface perturbations, however, are not visible before the electroporation pulse is delivered (Fig. 1a). In addition, these structures are no longer visible after the cell is allowed to recover from the pulse in BSK II medium for 30 min (data not shown).

We electroporated $B$. burgdorferi strain B31 with oligonucleotides encoding a mutation in the gyrB gene that confers coumermycin $A_{1}$ resistance in three separate experiments (Table 1). The transformation efficiency of the oligonucleotides was considerably lower than that obtained using the $840 \mathrm{bp}$ PCR-generated substrates, which typically yield about 20000 coumermycin $\mathrm{A}_{1}$ resistant colonies per $10 \mu \mathrm{g}$ of input DNA (2000 transformants per electroporation reaction) (Samuels et al., 1994a). In experiment 2, competent cells were frozen and stored at $-70^{\circ} \mathrm{C}$ before electroporation. This treatment lowers the transformation efficiency with PCR-amplified substrates by less than twofold. In all of the experiments, the 411R/398A399A oligonucleotide, which is the inverse complement of the coding strand (antisense), resulted in more transformants than the 385F/398T399T oligonucleotide (Table 1). Although the effect was small, mixing the two oligonucleotides immediately prior to electroporation resulted in a lower transformation efficiency than using either 411R/

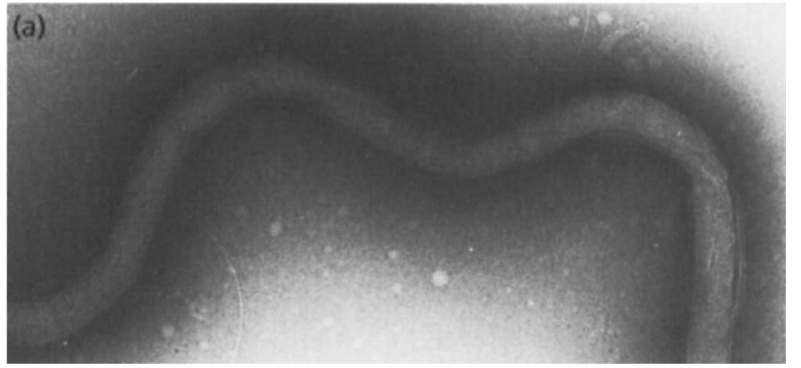

(b)

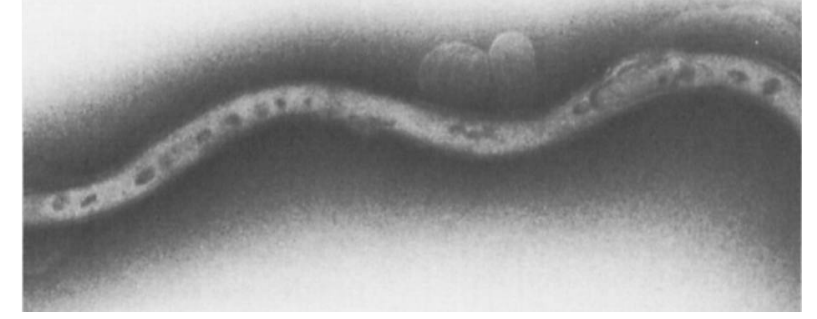

Fig. 1. Electron micrograph of $B$. burgdorferi before and after electroporation. Cells were washed extensively and either not subjected (a) or subjected (b) to an electrical pulse, followed by negative staining and observation by transmission electron microscopy. Periplasmic flagella were exposed or liberated during preparation of competent cells, which probably disrupted the outer membrane. In addition, membranous vesicles (blebs) are occasionally seen both free in the background and associated with spirochaetes (both electroporated and not electroporated).

398A399A by itself or a double-stranded oligonucleotide (Table 1), which suggests that the 385F/398T399T oligonucleotide is slightly inhibitory.

The spontaneous rate of mutation was two or fewer coumermycin $\mathrm{A}_{1}$-resistant colonies per electroporation reaction, as assessed using wild-type DNA sequences and irrelevant oligonucleotides as electroporation substrates (data not shown). To test if the coumermycin $A_{1}$ resistant colonies represented genuine transformants, DNA was isolated and sequenced from five colonies electroporated with 411R/398A399A in experiment 1 . Spontaneous mutants would be expected to have a single $G$ to $T$ transversion changing the Arg-133 AGA codon in $g y r B$ to an ATA Ile codon, while transformants would have a double mutation changing the Arg-133 AGA codon to an ATT Ile codon (Samuels et al., 1994a). All five coumermycin $\mathrm{A}_{1}$-resistant clones contained the double (site-directed) mutant ATT Ile codon, demonstrating that they resulted from successful transformation.

A heterologous strain, HB19, was also transformed and the results were qualitatively similar to that of strain B31, with the antisense oligonucleotide yielding the most transformants and the sense oligonucleotide demonstrating inhibitory activity (data not shown). However, compared to B31, the transformation efficiency of HB19 was about fivefold less using oligonucleotides and 10- to 100 -fold lower using a PCR product (amplified 
Table 1. Transformation efficiency of oligonucleotides

\begin{tabular}{|lccc|}
\hline & \multicolumn{3}{c|}{ Coumermycin A $_{1}$-resistant colonies per 10 $\boldsymbol{\mu g}$ DNA } \\
\cline { 2 - 4 } Oligonucleotide & Experiment 1 & Experiment 2 $\dagger$ & Experiment 3 \\
& & & \\
\hline 385F/398T399T & $<20$ & 18 & 27 \\
411R/398A399A & 350 & 52 & 150 \\
Double-stranded & ND & 24 & 70 \\
Mixed & ND & 6 & 10 \\
\hline
\end{tabular}

* ND, not done.

† Competent cells were frozen prior to electroporation.

from coumermycin $\mathrm{A}_{1}$-resistant B31). Five coumermycin $\mathrm{A}_{1}$-resistant clones from the PCR product electroporation and five from the 411R/398A399A oligonucleotide electroporation were isolated and their $\operatorname{gyr} B$ genes were partially sequenced. All 10 contained the marker mutation as described above, indicating that strain HB19 can be transformed with either oligonucleotides or PCR-generated substrates.

\section{DISCUSSION}

The effects of electroporation on the ultrastructure of $B$. burgdorferi are profound as judged by negative staining and electron microscopy. We believe that these micrographs represent the first visualization of electroporated bacteria. Darkly staining regions appear on the surface of the spirochaetes subjected to electroporation, but not on control cells. We suspect that these regions represent pores, induced by the electrical pulse, through which DNA can enter the cell (Nickoloff, 1995; Shigekawa \& Dower, 1988; Trevors et al., 1992).

The method presented here for the introduction of sitedirected mutations in vivo is both simple and convenient. Although the transformation efficiency is low, all of the isolated clones tested contained the mutations encoded by the oligonucleotide. We suspect that the transformation efficiency will increase with oligonucleotide length, as this would allow more target sequence for homologous recombination. Oligonucleotides as small as 20 bases can transform yeast, although transformation is optimal with 50 - to 60-base oligonucleotides (Moerschell et al., 1988). There were $\sim 1000$ times more oligonucleotide molecules than PCR-generated molecules per electroporation reaction in our experiments. Therefore the 840 bp PCR product results in $\sim 10000$ fold more transformants, on a molar basis, than the 27 base oligonucleotide.

The antisense oligonucleotide (411R/398A399A) produced 3 - to $>30$-fold more transformants per molecule than the sense oligonucleotide $(385 \mathrm{~F} /$ 398T399T). We do not know what mechanism may be responsible for this phenomenon, but the opposite preference occurs in yeast, with the sense oligonucleotide resulting in 20 -fold more transformants than the anti- sense oligonucleotide (Yamamoto et al., 1992). This implies that if interference with transcription by binding of the sense oligonucleotide to the mRNA occurs in $B$. burgdorferi, it is not a general phenomenon. The mixing experiment suggests that the sense oligonucleotide inhibited transformation in B. burgdorferi, while the antisense oligonucleotide did not inhibit transformation in yeast (Yamamoto et al., 1992). Therefore, different mechanisms may be responsible for the different strand preferences in the two organisms. Another possibility for the difference between the transformation efficiencies of the two oligonucleotides in B. burgdorferi is that purines are mismatched (G/A and $A / A)$ with the antisense oligonucleotide, while pyrimidines are mismatched $(\mathrm{T} / \mathrm{C}$ and $\mathrm{T} / \mathrm{T})$ with the sense oligonucleotide during recombination. The antisense oligonucleotide, which encodes the reverse complement of an Ile codon ( $3^{\prime}$ TAA $5^{\prime}$ ), hybridizes with the wild-type chromosomal sense strand, which carries an Arg codon ( $5^{\prime}$ AGA $\left.3^{\prime}\right)$, and the sense oligonucleotide ( $5^{\prime}$ ATT $\left.3^{\prime}\right)$ hybridizes with the wild-type chromosomal antisense strand $\left(3^{\prime}\right.$ TCT $5^{\prime}$ ). The homologous recombination system in Borrelia may prefer purine mismatches, which are bulkier. A third explanation is that the ends of the two oligonucleotides, which are different $(\mathrm{C}$ and $\mathrm{A}$ for the antisense and $G$ and $T$ for the sense oligonucleotide), or overall structure, contribute to either their capability to enter cells, stability in cells, or ability to recombine into the chromosome.

Strain HB19 has a lower transformation efficiency than strain B31, although there is a greater difference in transformation efficiencies between B31 (the source of the transformation substrate) and HB19 using the larger PCR-generated substrate compared to using the oligonucleotides. Besides the predicted mutations conferring coumermycin $A_{1}$ resistance, there are two nucleotide differences between the $840 \mathrm{bp}$ region of the PCRgenerated substrate from $\mathrm{B} 31$ and the homologous 840 bp region of the HB19 gyrB gene (data not shown). These two point mutations, which are outside of the sequence encoded by the oligonucleotides, may decrease transformation efficiency of the PCR product because mismatch repair could be initiated at more than one site after formation of the heteroduplex and a lethal double strand break might form if the excision tracts overlap. 
We plan to use oligonucleotide electroporation to assess the ability of other site-directed mutations in $\mathrm{gyr} B$ to confer coumarin resistance in B. burgdorferi, but the technique may be generally useful for introducing mutations into other genes or into other bacteria. If a convenient positive selection for a phenotype is available, then this method of site-directed mutagenesis is simple, straightforward and inexpensive.

\section{ACKNOWLEDGEMENTS}

We thank Mike Minnick, Kit Tilly and Eldon Walker for thoughtful and critical review of the manuscript, Stuart Hill (genetic recombination maven), Iain Old, David Persing (who suggested the use of oligonucleotides as transformation substrates) and Kit Tilly for valuable discussions, Van Tamplin for indispensable technical assistance, Joan Strange for proficient DNA sequencing (of the gyrB gene from HB19), Sandra Morrison for oligonucleotide synthesis, Bob Evans and Gary Hettrick for figure preparation and Dianne Huhtanen for medium production.

\section{REFERENCES}

Baranton, G. \& Old, I. G. (1995). The Spirochaetes: a different way of life. Bull Inst Pasteur 93, 63-95.

Barbour, A. G. \& Fish, D. (1993). The biological and social phenomenon of Lyme disease. Science 260, 1610-1616.

Hinnebusch, J. \& Tilly, K. (1993). Linear plasmids and chromosomes in bacteria. Mol Microbiol 10, 917-922.

Maxwell, A. (1993). The interaction between coumarin drugs and DNA gyrase. Mol Microbiol 9, 681-686.

Moerschell, R. P., Tsunasawa, S. \& Sherman, F. (1988). Transformation of yeast with synthetic oligonucleotides. Proc Natl Acad Sci USA 85, 524-528.

Nickoloff, J. A. (editor) (1995). Electroporation protocols for microorganisms. In Methods in Molecular Biology, p. 372. Series edited by J. M. Walker. Totowa, New Jersey: Humana Press.

Rosa, P. A. \& Hogan, D. M. (1992). Colony formation by Borrelia burgdorferi in solid medium: clonal analysis of osp locus variants. In First International Conference on Tick-Borne Pathogens at the Host-Vector Interface: An Agenda for Research, pp. 95-103.
Edited by U. G. Munderloh \& T. J. Kurtti. St Paul: University of Minnesota.

Rosa, P., Samuels, D. S., Hogan, D., Stevenson, B., Casjens, S. \& Tilly, K. (1996). Directed insertion of a selectable marker into a circular plasmid of Borrelia burgdorferi. J Bacteriol 178, 5946-5953.

Saint Girons, I., Old, I. G. \& Davidson, B. E. (1994). Molecular biology of the Borrelia, bacteria with linear replicons. Microbiology 140, 1803-1816.

Samuels, D. S. (1995). Electrotransformation of the spirochete Borrelia burgdorferi. In Electroporation Protocols for Microorganisms, pp. 253-259. Edited by J. A. Nickoloff. Totowa, New Jersey: Humana Press.

Samuels, D. S. \& Garon, C. F. (1993). Coumermycin A inhibits growth and induces relaxation of supercoiled plasmids in Borrelia burgdorferi, the Lyme disease agent. Antimicrob Agents Chemother 37, 46-50.

Samuels, D. S., Mach, K. E. \& Garon, C. F. (1994a). Genetic transformation of the Lyme disease agent Borrelia burgdorferi with coumarin-resistant gyrB. J Bacteriol 176, 6045-6049.

Samuels, D. S., Marconi, R. T., Huang, W. M. \& Garon, C. F. (1994b). gyrB mutations in coumermycin $A_{1}$-resistant Borrelia burgdorferi. J Bacteriol 176, 3072-3075.

Shigekawa, K. \& Dower, W. J. (1988). Electroporation of eukaryotes and prokaryotes: a general approach to the introduction of macromolecules into cells. BioTechniques 6, 742-751.

Steere, A. C. (1994). Lyme disease: a growing threat to urban populations. Proc Natl Acad Sci USA 91, 2378-2383.

Trevors, J. T., Chassy, B. M., Dower, W. J. \& Blaschek, H. P. (1992). Electrotransformation of bacteria by plasmid DNA. In Guide to Electroporation and Electrofusion, pp. 265-290. Edited by D. C. Chang, B. M. Chassy, J. A. Saunders \& A. E. Sowers. San Diego: Academic Press.

Yamamoto, T., Moerschell, R. P., Wakem, L. P., Komar-Panicucci, S. \& Sherman, F. (1992). Strand-specificity in the transformation of yeast with synthetic oligonucleotides. Genetics 131, 811-819.

Received 20 August 1996; revised 7 October 1996; accepted 10 October 1996. 